\title{
Chest Wall Pain, CTCAE 5.0
}

National Cancer Institute

\section{Source}

National Cancer Institute. Chest Wall Pain, CTCAE 5.0. NCI Thesaurus. Code C146743.

A disorder characterized by a sensation of marked discomfort in the chest wall. 